\title{
Can Early Diagnosis of Varices, Regular Praziquantel, and Reduction of Hepatitis Coinfection Reduce Mortality among Patients Attended for Periportal Fibrosis in Northwestern Tanzania? A Case-Control Study
}

\author{
Daniel W. Gunda $\mathbb{D}^{1,2}$ Elizabeth F. Mtui, ${ }^{1}$ Semvua B. Kilonzo $\left(\mathbb{D},{ }^{1,2}\right.$ Paulina M. Manyiri, \\ David C. Majinge, ${ }^{2}$ and Humphrey D. Mazigo ${ }^{3}$ \\ ${ }^{1}$ Department of Medicine, Catholic University of Health and Allied Sciences, 1464 Mwanza, Tanzania \\ ${ }^{2}$ Department of Medicine, Bugando Medical Centre, 1370 Mwanza, Tanzania \\ ${ }^{3}$ Department of Parasitology, Catholic University of Health and Allied Sciences, 1464 Mwanza, Tanzania
}

Correspondence should be addressed to Daniel W. Gunda; daniel_rev2002@yahoo.com

Received 21 November 2019; Accepted 28 February 2020; Published 13 March 2020

Academic Editor: José F. Silveira

Copyright (c) 2020 Daniel W. Gunda et al. This is an open access article distributed under the Creative Commons Attribution License, which permits unrestricted use, distribution, and reproduction in any medium, provided the original work is properly cited.

\begin{abstract}
Background. Schistosoma mansoni is highly endemic in the Lake Zone part of Tanzania and most people are chronically infected. Periportal fibrosis (PPF) is the commonest complication of chronic S. mansoni infection documented in up to $42 \%$ of studied participants in the community-based studies. These patients are at high risk of mortality since most of them are diagnosed late with bleeding varices. At Bugando, Schistosoma-related varices contributed to $70 \%$ of patients admitted due to vomiting blood with a two months' mortality of over $10 \%$. Earlier studies had reported higher mortality of up to $29 \%$ among patients with PPF even with the best in-hospital care. Understanding factors that increased the risk of mortality is important clinically in devising ways that can improve the outcome of this subgroup of patients. Methods. A retrospective analysis of patients with PPF from 2015 through 2018 was done. Their sociodemographic, clinical, laboratory, ultrasonographic, endoscopic, and survival status data were collected for analysis. STATA 13 was used for analysis, the prevalence of varices, active schistosomiasis, and hepatitis B coinfection was determined. Cumulative mortality as a major outcome was also determined, and factors associated with increased risk of mortality were assessed by a logistic regression model. Results. In total, 250 participants were included in this analysis. Majority, 222 (88.8\%; 95\% CI: 84.2-92.4) had active S. mansoni infection, and 40 (16.0\%; 95\% CI: 11.6-21.1) had S. mansoni-HBV coinfection. Cumulatively, 39 (15.6\%; 95\% CI: 11.3-20.7) patients died, with most deaths, 31 (79.5\%; 95\% CI: 63.5-90.7) occurring within two years following the diagnosis of PPF ( chi $^{2}=6.3 ; p=0.012$ ). The odds of mortality were independently associated with fishing (OR: 10.8; 95\% CI: 2.2-52; $p=0.003$ ), upper gastro intestinal bleeding (OR: 2.4; 95\% CI: 1.1-5.4; $p=0.037$ ), HBV coinfection (OR: 3.3; 95\% CI: 1.2-91; $p=0.019$ ), and ascites (OR: 3.3; 95\% CI: $1.3-8.2 ; p=0.010$ ). Conclusions. In this, S. mansoni endemic area, varices, actives schistosomiasis, hepatitis B coinfection, and mortality are highly common. Screening for varices and initiation of prophylaxis, administration of praziquantel, and screening for hepatitis B should be part and parcel of care of these patients. The first two years of diagnosis, patients are at high risk of mortality; risk factors in this study should assist planning a closer follow-up of patients at risk of mortality to improve their long-term outcome.
\end{abstract}

\section{Background}

Schistosoma mansoni affects over 54 million people worldwide, and it is a major cause of high morbidity and mortality only second to malaria. More than 400 million people are at risk of infection especially in Sub-Saharan Africa (SSA) where more than $90 \%$ of the world's burden of $S$. mansoni is concentrated. Tanzania is the second most affected country after Nigeria [1], and S. mansoni is highly endemic in the Lake Zone part of the country especially among communities 
that are engaged in freshwater activities like fishing and rice farming $[2,3]$. Due to the nature of these breadwinning activities, most people in Tanzania are at risk of chronic S. mansoni infection with serious clinical complications. Periportal fibrosis (PPF) commonly complicates chronic S. mansoni infection with high mortality due to bleeding varices $[4,5]$.

Community-based studies indicated that at least $30 \%$ of people infected $S$. mansoni have associated PPF. In the lake zone of Tanzania, up to $42 \%$ of patients studied for S. mansoni infection have been shown to have associated PPF [6, 7]. Similarly, hospital-based studies have also demonstrated a higher encounter of attendant varices among patients with PPF with high mortality. For instance in Saudi, $72 \%$ of patients who had PPF were found to have associated esophageal varices [8] before incident bleeding. However, it is estimated that about $30 \%$ of patients with PPF present late enough with fatal bleeding varices ending up with a mortality of $10-20 \%$ which might be even higher $[9,10]$. A study from Bugando indicated that varices caused $70 \%$ of hematemesis with high mortality of more than $10 \%$ [10]. Studies elsewhere had reported even higher mortality rates up to $29 \%$ of PPF patients presenting with hematemesis $[11,12]$.

Previous studies had indicated that the mortality is increased among those patients who are coinfected with hepatitis B, severe liver fibrosis, and larger portal vein among others. Studies on factors associated with increased risk of mortality are still scarce especially in our setting where S. mansoni is still highly endemic. Understanding factors that increased the risk of mortality is important clinically in devising ways that can improve the outcome of this subgroup of patients.

\section{Material and Methods}

This was a clinic-based retrospective study involving all adult patients who were diagnosed to have periportal fibrosis between January 2015 and December 2018. The study was conducted at Bugando Medical Centre (BMC). BMC is a university teaching hospital that started operating under superspecialized units in 2015 with gastroenterology unit being one of the well-doing units in internal medicine. The unit has functioning endoscopic facilities and it works in collaboration with other departments including radiology and the main laboratory.

Patients with periportal fibrosis and cirrhosis are attended on daily bases in gastroenterology unit as common causes of portal hypertension. Routinely, abdominal ultrasound examination is done to determine the probable cause the portal hypertension and other details including portal vein and splenic diameter, and presence or absence of ascites is documented. Subsequently, endoscopic screening for attendant esophageal varices is done, and other additional tests including full blood count (FBP), hepatitis B surface antigen, liver function tests (LFT), and urine CCA or stool for $S$. mansoni are also done. Patients with PPF due to $S$. mansoni routinely get biannual praziquantel; propranolol for those with small varices and a series of endoscopic variceal ligation (EVL) in addition to propranolol is done among those with large varices.
A minimum sample size of 246 patients was estimated from the Kish-Lisle formula assuming cumulative mortality of 20\% among patients with PPF as reported previously [9]. A clinic registry was used to identify all patients who were diagnosed to have PPF at BMC during the study period. Registration numbers were used to trace patients' files which were then reviewed by researchers for information of research interest. Demographic information, clinical presentation including abdominal distension, hematemesis, and passage of bloody stools; UTS details including splenic size, portal vein diameter (PVD), and ascites; test results for $S$. mansoni, FBP, AST, and ALT; upper endoscopy results; and survival status were recorded for analysis. Time in years from diagnosis of PPF to the enrolment into the study was recorded in years, and for patients who were dead, the time in years from diagnosis to death was noted.

Data were computerized using Epi data version 3.1, and STATA version 13 (Stata Corp LP, college station, TX) was used for analysis. Continuous variables were summarized as medians with interquartile range (IQR) while categorical variables were summarized as proportions with percentages. Death as the main outcome of patients was calculated and expressed as a percentage with $95 \%$ confidence interval (CI) and the distribution of deaths by time from diagnosis of PPF was determined. Odds ratio with 95\% CI was calculated by univariate followed by a multivariate logistic regression model to assess the difference in degree of association between various factors with the mortality outcome. All factors with $p<0.25$ on the univariate model were considered for subsequent inclusion in the multivariate model. In the final model factors with a $p$ value $<0.05$ were considered to have an independent association with the mortality outcome and the assessment for fitness of the model was done by Hosmer-Lemeshow.

2.1. Ethical Clearance. The permission to conduct and publish the findings from this study was sought from the Catholic University of Health and Allied Sciences (CUHAS)/BMC joint ethical committee with an ethical clearance certificate number 907/2019. The patients' information was handled by the researcher alone and their identifiers including names and registration numbers were not included in the final analysis to further maintain confidentiality.

\section{Results}

3.1. Sociodemographic, Clinical, and Laboratory Characteristics among 250 Participants. In total, 250 participants with $S$. mansoni-related PPF were analyzed. The majority, $180(72 \%)$, were male participants with a median age of 41 (IQR: 35-51) years and a median postdiagnosis time of 2 (IQR: 1-3) years. Most participants, 197 (78.8\%), were peasants and $24(9.6 \%)$ were still engaging in fishing activities; however, the majority, 238 (95.6\%), had contact with Lake Victoria water. Most patients, 171 (68.4\%), presented with abdominal distension and $64(25.6 \%)$ had a history of upper gastrointestinal bleeding. Of the studied participants, 108 (43.2\%; 95\% CI: 36.9-49.6\%) were found to have esophageal varices, and 222 (88.8\%; 95\% CI: 84.2-.92.4) had active 
TABle 1: Study characteristics among 250 participants with Schistosoma periportal fibrosis.

\begin{tabular}{|c|c|c|}
\hline Variable & Frequency & Percent (\%) or median (IQR) \\
\hline \multicolumn{3}{|l|}{ Sex } \\
\hline Male & 180 & 72.0 \\
\hline Female & 70 & 28.0 \\
\hline Age (years) & 250 & $41(33-51)$ \\
\hline \multicolumn{3}{|l|}{ Married } \\
\hline Yes & 215 & 86.0 \\
\hline No & 35 & 14.0 \\
\hline \multicolumn{3}{|l|}{ Occupation } \\
\hline Peasant & 197 & 78.8 \\
\hline Fishing & 24 & 9.6 \\
\hline Others & 29 & 11.6 \\
\hline \multicolumn{3}{|l|}{ Lake water contact } \\
\hline Yes & 238 & 95.6 \\
\hline No & 11 & 4.4 \\
\hline \multicolumn{3}{|l|}{ Alcohol use } \\
\hline Yes & 123 & 49.2 \\
\hline No & 127 & 50.8 \\
\hline \multicolumn{3}{|l|}{ Abdominal distension } \\
\hline Yes & 171 & 68.4 \\
\hline No & 79 & 31.6 \\
\hline \multicolumn{3}{|l|}{ Upper GIT bleeding } \\
\hline Yes & 64 & 25.6 \\
\hline No & 186 & 74.4 \\
\hline \multicolumn{3}{|l|}{ Varices on OGD } \\
\hline Yes & 108 & 43.2 \\
\hline No & 142 & 56.8 \\
\hline \multicolumn{3}{|l|}{ APRI $>1.5$} \\
\hline Yes & 90 & 36.0 \\
\hline No & 160 & 64.0 \\
\hline Years postdiagnosis & 250 & $2(1-3)$ \\
\hline
\end{tabular}

APRI: aspartate aminotransferase platelet count ratio Index; GIT: gastrointestinal tract; IQR: interquartile range; OGD: oesophagogastroduodenoscopy.

S. mansoni infection. In total, 44 (17.6\%; 95\% CI: 13.1-.22.9) had positive test for HBsAg and 40 (16.0\%; 95\% CI: 11.621.1) had S. mansoni-BV coinfection (Table 1). Patients with $S$. mansoni-hepatitis B coinfection were more likely to have a fibrosis APRI score $>1.5$ (52.5\% vs.32.4\%, chi ${ }^{2}$ : 5.6; $\operatorname{Pr}=0.018$ ) as summarized in (Table 2 and Figure 1).

3.2. Prevalence and Correlates of Mortality among 250 Study Participants with PPF. In this study, a total of 39 (15.6\%; 95\% CI: 11.3-20.7) were reported to pass on. Most of the reported deaths, 31(79.5\%; 95\% CI: 63.5-90.7), were more likely to occur within the first two years following diagnosis of PPF $\left(\mathrm{chi}^{2}=6.3 ; p=0.012\right)$ (Figure 2$)$. The odds of mortality were independently associated with fishing (OR: 10.8; 95\% CI: 2.2-52; $p=0.003)$, upper gastrointestinal bleeding (OR: 2.4 ; 95\% CI: 1.1-5.4; $p=0.037)$, S. mansoni-HBV coinfection (OR: 3.3; 95\% CI: 1.2-91; $p=0.019$ ), and ascites (OR:
TABLE 2: Assessment of liver fibrosis by APRI scores among 250 study participants.

\begin{tabular}{|c|c|c|c|c|}
\hline \multirow{2}{*}{ SHB coinfection } & \multicolumn{3}{|c|}{ AST to platelet ratio index class } & \multirow{2}{*}{ Total } \\
\hline & $\leq 0.5$ & $0.5-1.5$ & $\geq 1.5$ & \\
\hline No $(n \%)$ & $40(19.1)$ & $102(48.6)$ & $68(32.4)$ & $210(100.0)$ \\
\hline Yes $(n \%)$ & $2(5.0)$ & $17(42.5)$ & $21(52.5)$ & $40(100.0)$ \\
\hline Total $(n \%)$ & $42(16.8)$ & $119(47.6)$ & $89(35.6)$ & $250(100.0)$ \\
\hline
\end{tabular}

S. mansoni-HBV coinfection status and a liver fibrosis by APRI score

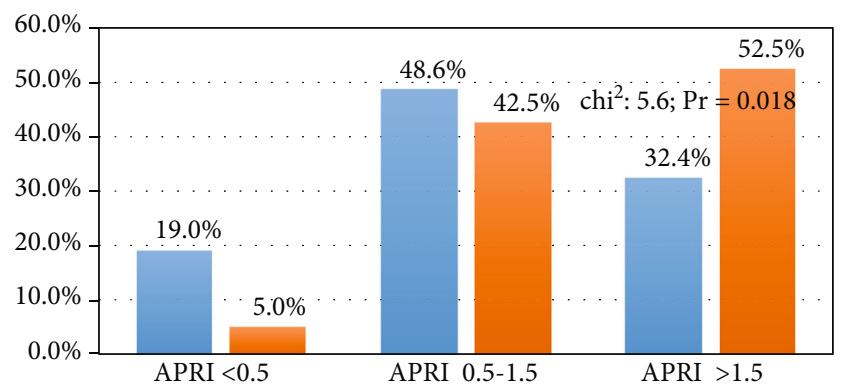

S. mansoni-HBV coinfection (-VE)

S. mansoni-HBV coinfection (+VE)

FIgURE 1: S. mansoni-HBV coinfection status and liver fibrosis by APRI score.

Distribution of deaths by years post diagnosis of periportal fibrosis

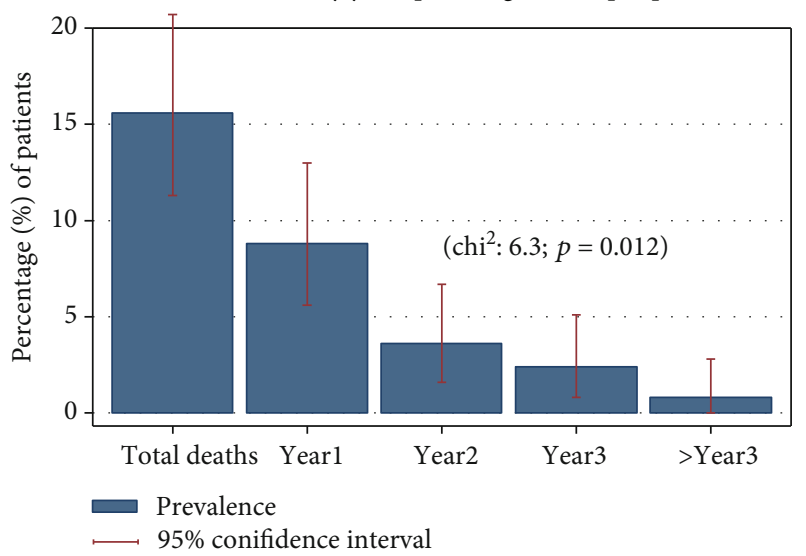

FIgure 2: Distribution of deaths by years post diagnosis of periportal fibrosis.

3.3; 95\% CI: $1.3-8.2 ; p=0.010)$. The difference in the distribution of other factors was not significant statically (Table 3), and the assessment for goodness of fit of the model did not demonstrate gross lack of fitness with area under the ROC curve of 0.8124 (Figure 3).

\section{Discussion}

The main objective of this study was to determine the prevalence and correlates of mortality among patients attended for 
TABLE 3: Factors associated with mortality among 250 patients with periportal fibrosis.

\begin{tabular}{|c|c|c|c|c|c|c|}
\hline \multirow{2}{*}{ Variable } & \multicolumn{2}{|c|}{ Mortality postdiagnosis } & \multicolumn{2}{|c|}{ Unadjusted } & \multicolumn{2}{|c|}{ Adjusted } \\
\hline & No $(n=211)$ & Yes $(n=39)$ & OR $(95 \% \mathrm{CI})$ & $p$ value & OR $(95 \% \mathrm{CI})$ & $p$ value \\
\hline \multicolumn{7}{|l|}{ Sex } \\
\hline Female & $63(29.9)$ & $7(18.0)$ & 1.0 & & & \\
\hline Male & $148(70.1)$ & $32(82.1)$ & $1.9(0.8-4.6)$ & 0.133 & $1.2(0.4-3.1)$ & 0.728 \\
\hline Age & $41(33-51)$ & $41(35-54)$ & $1.0(0.9-1.03)$ & 0.601 & & \\
\hline \multicolumn{7}{|c|}{ Lake water contact } \\
\hline No & $10(4.8)$ & $1(2.6)$ & 1.0 & & & \\
\hline Yes & $200(95.2)$ & $38(97.4)$ & $1.8(0.2-15.3)$ & 0.546 & & \\
\hline \multicolumn{7}{|l|}{ Occupation } \\
\hline Fishing & $13(6.2)$ & $11(28.2)$ & $5.9(2.4-14.6)$ & $<0.001$ & $10.8(2.2-52.0)$ & 0.003 \\
\hline Peasant & $173(82.0)$ & $24(61.5)$ & $0.3(0.2-0.7)$ & 0.005 & $1.1(0.3-4.1)$ & 0.844 \\
\hline \multicolumn{7}{|l|}{ Upper GIB } \\
\hline No & $163(77.3)$ & $23(58.0)$ & 1.0 & & & \\
\hline Yes & $48(22.7)$ & $16(41.0)$ & $2.4(1.1-4.8)$ & 0.018 & $2.4(1.1-5.4)$ & 0.037 \\
\hline \multicolumn{7}{|l|}{ S. mansoni } \\
\hline Negative & $20(9.5)$ & $8(20.5)$ & 1.0 & & & \\
\hline Positive & $191(90.5)$ & $31(79.5)$ & $0.4(0.2-1.0)$ & 0.050 & $0.2(0.1-0.6)$ & 0.005 \\
\hline \multicolumn{7}{|c|}{ HBV coinfection } \\
\hline No & $183(86.7)$ & $27(69.2)$ & 1.0 & & & \\
\hline Yes & $28(13.3)$ & $12(30.8)$ & $2.9(1.3-6.3)$ & 0.008 & $3.3(1.2-9.1)$ & 0.019 \\
\hline $\operatorname{PTC}\left(10^{3} / \mu \mathrm{l}\right)$ & $89(59-126)$ & $93(71-185)$ & $1.0(0.9-1.0)$ & 0.680 & & \\
\hline $\operatorname{PVD}(\mathrm{cm})$ & $1.5(1.4-1.7)$ & $1.5(1.4-1.8)$ & $1.8(0.8-4.1)$ & 0.117 & $2.4(0.7-8.4)$ & 0.145 \\
\hline APRI & $1.1(0.7-2.2)$ & $1.0(0.6-2.0)$ & $0.9(0.7-1.1)$ & 0.488 & & \\
\hline $\mathrm{SPD}(\mathrm{cm})$ & $17(15-18)$ & $17(15.19)$ & $1.1(0.9-1.2)$ & 0.289 & & \\
\hline \multicolumn{7}{|l|}{ Ascites } \\
\hline No & $101(47.9)$ & $9(23.1)$ & 1.0 & & & \\
\hline Yes & $110(52.1)$ & $30(76.9)$ & $3.1(1.3-6.7)$ & 0.006 & $3.3(1.3-8.2)$ & 0.010 \\
\hline \multicolumn{7}{|c|}{ Varices on OGD } \\
\hline No & $116(55.0$ & $25(64.1)$ & 1.0 & & & \\
\hline Yes & $95(45.0)$ & $14(35.9)$ & $0.6(0.3-1.3)$ & 0.293 & & \\
\hline
\end{tabular}

CI: confidence interval; HBV: hepatitis B virus; OR: odds ratio; PTC: platelet count; SPD: splenic diameter; PVD: portal vein diameter; UGIB: upper gastro intestinal bleeding.

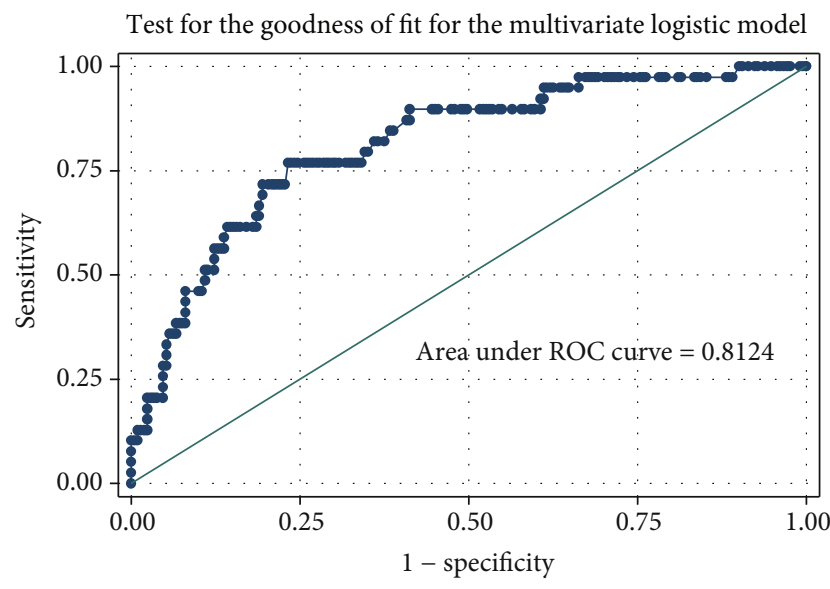

Figure 3: Test for the goodness of fit for the multivariate logistic model. periportal fibrosis at a tertiary level hospital in Mwanza Tanzania. In this study, 250 participants were analyzed where $108(43.2 \%)$ were endoscopically found to have esophageal varices, $222(88.8 \%)$ had active $S$. mansoni infection, and 44 $(17.6 \%)$ were coinfected with hepatitis B. Over a median period of 2 (IQR: 1-3) years, 39 (15.6\%) participants died and the risk of mortality was higher with fishing, hematemesis, ascites, and hepatitis B coinfection.

The prevalence of esophageal varices in our current study is similar to a prevalence rate of $45 \%$ reported from Uganda [13] and 47\% reported in Sudan before praziquantel (PZQ) mass drug administration (MDA) [14]. However, the current prevalence rate is lower than the prevalence of $67 \%$ reported much earlier in a study from Sudan [15] and $72 \%$ that was reported from Saudi in 2011 [8]. Comparatively, the prevalence of esophageal varices in the current study is higher than the prevalence rate of $30 \%$ in Sudan the following year of MDA which 
was also associated with a significant reduction of PPF severity and S. mansoni infection [14]. The difference in prevalence of varices observed is more likely due to a discrepancy in the timing of screening for varices in these studies.

In our setting, active $S$. mansoni among patients with periportal fibrosis seems to be a very common encounter. The prevalence of active $S$. mansoni in the current study is similar to a previous report from Zambia $(88.8 \%$ vs. $88 \%)$ [16]. However, it is slightly lower than earlier reports from Zambia ( $88.8 \%$ vs. $98 \%$ ) where the diagnosis of active S. mansoni was based on rectal snip for Schistosoma ova [17]. Otherwise, the current prevalence is comparatively higher than what was reported from Kenya (88.8\% vs. $72 \%$ ) [18], $60 \%$ reported in 2014 from Bugando [10], 59.0\% from Zimbabwe [19], and 53\% reported from Sudan [14]. The current prevalence of active $S$. mansoni also is much higher than a prevalence of rate $34.5 \%$ reported recently from Bugando Medical Centre among PPF patients undergoing endoscopic variceal ligation for large varices [20].

Ongoing lake contact can partly explain the higher prevalence of active $S$. mansoni reported from Zambia [16] which is also similar to our current study where $238(95.6 \%)$ of the studied participants had reported repeated contact with lake water. Moreover, the diagnosis of $S$. mansoni based on rectal snips could have included even those participants with nonviable ova in a study from Zambia [17]. Otherwise, PPF patients attending endoscopy unit for endoscopic variceal ligation are usually put on biannual praziquantel at Bugando [20], and probably those found with active $S$. mansoni infection represent a subgroup of patients with Schistosoma reinfection since this is a frequent phenomenon reported elsewhere following mass drug administration [21,22].

The prevalence of $S$. mansoni-hepatitis B coinfection in this study is similar to concurrent infection of $15.8 \%$ reported by Aquino et al. from Brazil [23] and 16.1\% reported in another study done by Berhe et al. in Ethiopia [24]. However the prevalence of $S$. mansoni and hepatitis B coinfection in the current study is comparatively lower than the prevalence rate of $19.6 \%$ reported earlier in 1991 by El-Sayed et al. from Egypt [25] and 58.4\% reported recently by Du et al. from China [26]. But even with these difference on clinical grounds, this indicates that the Schistosoma endemic areas are also endemic for hepatitis B whose coinfection has been shown to have an even much severe liver disease and poorer outcome [27].

With the mortality rate reported in this study, 39 (15.6\%) are comparatively similar to a previous study from Bugando among 124 patients with hematemesis. In this study, 91 participants underwent endoscopy and 13 (14.3\%) died within 2 months of follow-up [10]. Otherwise the current mortality rate is higher than what was reported by Kheir et al. from Sudan $(16.0 \%$ vs. $11.0 \%)$ in 2000 [14], and it is also much higher than the mortality of $6.25 \%$ among patients with periportal fibrosis who had rebleeding [28] and 5.9\% reported from Uganda in 2007 [29]. Earlier studies from Sudan had reported a much higher prevalence of mortality of $25-29 \%$ following bleeding events $[11,12]$.
Mortality in this subgroup of patients partly denotes a delayed diagnosis of attendant varices and subsequent prevention of bleeding [30]. This is well supported in the current study that bleeding was reported in nearly $60 \%(n=64)$ out of 108 of those with varices and the mortality was 2.4 times more likely among those with bleeding as compared to the nonbleeding counterparts $(41.0 \%$ vs. $22.7 \%$, OR: $2.4 ; p=$ $0.037)$. This is likely to occur among those with recurrent $S$. mansoni infection, in this case, those who are occupationally exposed to lake water [10] like the fishermen in this study. In previous studies, these patients were more likely to have severe periportal fibrosis on ultrasound assessment with a large portal vein diameter. But they were also more likely to have ascites similar to findings in our current study [10, 31-33] among others.

Coinfection with hepatitis B has been associated with higher odds of mortality previously in this subgroup of patients [31], similar to the findings in the current study. In an earlier study by Bassily et al., it was indicated that the mortality rate among $S$. mansoni-hepatitis B-coinfected patients was as high as $64.0 \%$ vs. $22 \%$ among those who were monoinfected [34]. This has been established previously as being due to severe inflammatory process with severe liver damage that occurs among patients who are S. mansoni-hepatitis Bcoinfected patients which have also been shown to end up with increased morbidity and mortality $[27,35]$. This is also supported partly by the finding that patients who were $S$. mansoni-hepatitis B coinfected were more likely to have higher fibrosis APRI scores of $>1.5$ as compared to their noncoinfected counterparts.

These findings are clinically important in our setting where S. mansoni is highly endemic suggesting that at any time, patients with PPF are at greater risk of having an ongoing active Schistosoma infection due to a possible high rate of reinfection. So apart from biannual PZQ, screening and treatment for active $S$. mansoni infection are advocated on routine bases. Giving PZQ is shown to reverse PPF and potentially reduces mortality [36, 37]. Routine screening for hepatitis B coinfection also is suggested by findings in this study. Patients with coinfection are likely to have a worse prognosis as compared to those who are monoinfected. Otherwise, patients with PPF should have an endoscopy done $[32,38]$ and prophylaxis started, with a close clinical follow-up particularly in the first two years [38]. Risk factors in this study are useful in the selection of patients at risk of mortality and on closer clinical follow-up to improve their outcomes.

This study is also liable to several limitations. It is a single-center study; thus, its findings may not be generable. Being retrospective, it lacks the temporal relation of events and a longitudinal assessment of the effect of treatment, and thus, a longitudinal study is recommended to address some of these issues. Otherwise, the information from this study is highly valuable in plans to address this important subgroup of people.

\section{Data Availability}

No data were used to support this study. 


\section{Ethical Approval}

Ethical clearance was obtained with certificate no 907/2019, consent to participate not applicable.

\section{Conflicts of Interest}

The authors declare to have no competing interest to declare.

\section{Authors' Contributions}

DWG, HDM, and SBK participated in conception and designing of the study. EFM, DCM, and PMM acquired the data. DWG, HDM, and SBK did data analysis and interpretation. DWG did manuscript drafting. All the authors critically reviewed the manuscript for its intellectual content and approved the final version.

\section{Acknowledgments}

The authors would like to acknowledge the great support given by the Department of Medical Records at BMC during retrieval of patients' information.

\section{References}

[1] WHO, Schistosomiasis: Progress Report 2001-2011 and Strategic Plan 2012-2020, World Health Organization, 2013.

[2] S. Brooker, N. B. Kabatereine, J. L. Smith et al., "An updated atlas of human helminth infections: the example of East Africa," International Journal of Health Geographics, vol. 8, no. 1, p. 42, 2009.

[3] P. Steinmann, J. Keiser, R. Bos, M. Tanner, and J. Utzinger, "Schistosomiasis and water resources development: systematic review, meta-analysis, and estimates of people at risk," The Lancet Infectious Diseases, vol. 6, no. 7, pp. 411-425, 2006.

[4] C. K. Opio, F. Kazibwe, P. Ocama, L. Rejani, E. N. Belousova, and P. Ajal, "Profiling lifetime episodes of upper gastrointestinal bleeding among patients from rural Sub-Saharan Africa where Schistosoma mansoni is endemic," The Pan African Medical Journal, vol. 24, 2016.

[5] D. J. Gray, A. G. Ross, Y. S. Li, and D. P. McManus, "Diagnosis and management of schistosomiasis," BMJ, vol. 342, article d2651, 2011.

[6] R. Kardorff, R. M. Gabone, C. Mugashe et al., "Schistosoma mansoni-related morbidity on Ukerewe Island, Tanzania: clinical, ultrasonographical and biochemical parameters," Tropical Medicine \& International Health, vol. 2, no. 3, pp. 230-239, 1997.

[7] H. D. Mazigo, F. Nuwaha, D. W. Dunne et al., "Schistosoma mansoni infection and its related morbidity among adults living in selected villages of Mara Region, North-Western Tanzania: a cross-sectional exploratory study," The Korean Journal of Parasitology, vol. 55, no. 5, pp. 533-540, 2017.

[8] A. Agha, M. M. Abdulhadi, S. Marenco et al., "Use of the platelet count/spleen diameter ratio for the noninvasive diagnosis of esophageal varices in patients with schistosomiasis," Saudi Journal of Gastroenterology, vol. 17, no. 5, pp. 307-311, 2011.

[9] F. F. Makdissi, P. Herman, V. Pugliese et al., "Long-term results of esophagogastric devascularization and splenectomy associated with endoscopic treatment in schistosomal portal hypertension," World Journal of Surgery, vol. 34, no. 11, pp. 2682-2688, 2010.

[10] A. A. Chofle, H. Jaka, M. Koy et al., "Oesophageal varices, schistosomiasis, and mortality among patients admitted with haematemesis in Mwanza, Tanzania: a prospective cohort study," BMC Infectious Diseases, vol. 14, no. 1, p. 303, 2014.

[11] S. Bessa and I. Helmy, "Injection sclerotherapy for esophageal varices caused by schistosomal hepatic fibrosis," Surgery, vol. 97, no. 2, pp. 164-168, 1985.

[12] A. El-Zayadi, S. S. El-Din, and S. Kabil, "Endoscopic sclerotherapy versus medical treatment for bleeding esophageal varices in patients with schistosomal liver disease," Gastrointestinal Endoscopy, vol. 34, no. 4, pp. 314-317, 1988.

[13] M. Ravera, A. Reggiori, E. Cocozza, F. Ciantia, and G. Riccioni, "Clinical and endoscopic aspects of hepatosplenic schistosomiasis in Uganda," European Journal of Gastroenterology \& Hepatology, vol. 8, no. 7, pp. 693-697, 1996.

[14] M. M. Kheir, O. Z. Baraka, I. A. el-Tom, M. M. Mukhtar, and M. M. Homieda, "Effects of single-dose praziquantel on morbidity and mortality resulting from intestinal schistosomiasis," Eastern Mediterranean Health Journal, vol. 6, no. 5-6, pp. 926931, 2000 .

[15] A. M. Saad, M. Homeida, I. Eltom, T. Nash, J. L. Bennett, and M. A. Hassan, "Oesophageal varices in a region of the Sudan endemic for Schistosoma mansoni," The British Journal of Surgery, vol. 78, no. 10, pp. 1252-1253, 1991.

[16] L. Payne, E. Turner-Moss, M. Mutengo, A. W. Asombang, and P. Kelly, "Prevalence of schistosome antibodies with hepatosplenic signs and symptoms among patients from Kaoma, Western Province, Zambia," BMC Research Notes, vol. 6, no. 1, 2013.

[17] R. N. Davidson, S. Houston, and C. F. Kiire, "Schistosomal periportal fibrosis in Zimbabwe: use of ultrasound in patients with oesophageal varices," Transactions of the Royal Society of Tropical Medicine and Hygiene, vol. 85, no. 3, pp. 380382, 1991.

[18] K. M. De Cock, S. Awadh, R. S. Raja, R. Jupp, B. M. Wankya, and S. B. Lucas, "Portal hypertension in Nairobi, Kenya," Bulletin de la Société de pathologie exotique et de ses filiales, vol. 76, no. 5, pp. 567-570, 1983.

[19] S. Houston, M. Munjoma, K. Kanyimo, R. N. Davidson, and G. Flowerdew, "Use of ultrasound in a study of schistosomal periportal fibrosis in rural Zimbabwe," Acta Tropica, vol. 53, no. 1, pp. 51-58, 1993.

[20] H. D. Mazigo, O. Suleiman, P. Manyiri, D. Ruganuza, D. W. Gunda, and D. C. Majinge, "Praziquantel treatment is recommended: active schistosoma mansoni infection among patients diagnosed with oesophageal varices at a Tertiary Referral Hospital, North-Western Tanzania," Annals of Short Reports, vol. 2, article 1035, 2019.

[21] E. Woldegerima, A. G. Bayih, Y. Tegegne, M. Aemero, and A. Jejaw Zeleke, "Prevalence and reinfection rates of Schistosoma mansoni and praziquantel efficacy against the parasite among primary school children in Sanja Town, Northwest Ethiopia," Journal of Parasitology Research, vol. 2019, Article ID 3697216, 8 pages, 2019.

[22] E. C. Mbanefo, N. T. Huy, A. A. Wadagni, C. I. Eneanya, O. Nwaorgu, and K. Hirayama, "Host determinants of reinfection with schistosomes in humans: a systematic review and meta-analysis," PLoS Neglected Tropical Diseases, vol. 8, no. 9, article e3164, 2014. 
[23] R. T. Aquino, P. P. Chieffi, S. M. Catunda et al., "Hepatitis B and $\mathrm{C}$ virus markers among patients with hepatosplenic mansonic schistosomiasis," Revista do Instituto de Medicina Tropical de São Paulo, vol. 42, no. 6, pp. 313-320, 2000.

[24] N. Berhe, B. Myrvang, and S. G. Gundersen, "Intensity of Schistosoma mansoni, hepatitis B, age, and sex predict levels of hepatic periportal thickening/fibrosis (PPT/F): a largescale community-based study in Ethiopia," The American Journal of Tropical Medicine and Hygiene, vol. 77, no. 6, pp. 1079-1086, 2007.

[25] H. F. El-Sayed, S. M. Abaza, S. Mehanna, and P. J. Winch, "The prevalence of hepatitis $\mathrm{B}$ and $\mathrm{C}$ infections among immigrants to a newly reclaimed area endemic for Schistosoma mansoni in Sinai, Egypt," Acta Tropica, vol. 68, no. 2, pp. 229-237, 1997.

[26] H. Du, "Relationship between advanced schistosomiasis and HBV infection," Zhongguo Xue Xi Chong Bing Fang Zhi Za Zhi, vol. 25, no. 1, pp. 115-117, 2013.

[27] H. H. Omar, "Impact of chronic schistosomiasis and HBV/HCV co-infection on the liver: current perspectives," Hepatic Medicine: Evidence and Research, vol. 11, pp. 131136, 2019.

[28] I. Mostafa, M. M. Omar, and A. Nouh, "Endoscopic control of gastric variceal bleeding with butyl cyanoacrylate in patients with schistosomiasis," Journal of the Egyptian Society of Parasitology, vol. 27, no. 2, pp. 405-410, 1997.

[29] R. Maurizio, C. Eugenio, and P. R. Roberto, "Results of sclerotherapy for bleeding esophageal varices in patients with schistosomal liver disease. A retrospective study," HepatoGastroenterology, vol. 47, no. 32, pp. 424-428, 2000.

[30] K. M. de Cock, S. Awadh, S. B. Lucas, R. S. Raja, and B. M. Wankya, "Esophageal varices in Nairobi, Kenya: a study of 68 cases," The American Journal of Tropical Medicine and Hygiene, vol. 31, no. 3, pp. 579-588, 1982.

[31] M. A. Madwar, M. K. Shaker, M. A. Atta, T. H. el Khashaab, and M. K. Mohamed, "A prospective study: prediction of the first variceal hemorrhage in schistosomal and nonschistosomal liver disease," The Journal of the Egyptian Public Health Association, vol. 72, no. 3-4, pp. 395-409, 1997.

[32] J. Richter, E. da Silva Monteiro, R. Moreira Braz et al., "Sonographic organometry in Brazilian and Sudanese patients with hepatosplenic schistosomiasis mansoni and its relation to the risk of bleeding from oesophageal varices," Acta Tropica, vol. 51, no. 3-4, pp. 281-290, 1992.

[33] M. A. Hassan, A. M. Abass, H. O. Mirghani, E. Osman, and M. O. E. Gadour, "Noninvasive prediction of esophageal varices grade (size) in Sudanese patients with periportal fibrosis," International Journal of Gastroenterology, vol. 2, no. 2, pp. 28-33, 2018.

[34] S. Bassily, Z. Farid, G. I. Higashi, I. A. Kamel, N. A. el-Masry, and R. H. Watten, "Chronic hepatitis B antigenaemia in patients with hepatosplenic schistosomiasis," The Journal of Tropical Medicine and Hygiene, vol. 82, no. 11-12, pp. 248251, 1979.

[35] L. G. Lyra, G. Reboucas, and Z. A. Andrade, "Hepatitis B surface antigen carrier state in hepatosplenic schistosomiasis," Gastroenterology, vol. 71, no. 4, pp. 641-645, 1976.

[36] N. M. El-Lakkany, O. A. Hammam, W. H. El-Maadawy, A. A. Badawy, A. A. Ain-Shoka, and F. A. Ebeid, "Anti-inflammatory/anti-fibrotic effects of the hepatoprotective silymarin and the schistosomicide praziquantel against Schistosoma mansoni-induced liver fibrosis," Parasites \& Vectors, vol. 5, no. $1,2012$.
[37] P. Martins-Leite, G. Gazzinelli, L. F. Alves-Oliveira et al., "Effect of chemotherapy with praziquantel on the production of cytokines and morbidity associated with schistosomiasis mansoni," Antimicrobial Agents and Chemotherapy, vol. 52, no. 8, pp. $2780-2786,2008$.

[38] H. M. Y. Mudawi and K. B. Ibrahim, "Endoscopic variceal sclerotherapy in patients with Symmers periportal fibroses," Tropical Doctor, vol. 37, no. 3, pp. 179-181, 2016. 